\title{
Spatially-Coupled MacKay-Neal Codes with No Bit Nodes of Degree Two Achieve the Capacity of BEC
}

\author{
Takuya Okazaki and Kenta Kasai \\ Department of Communications and Computer Engineering, \\ Tokyo Institution of Technology \\ Email: \{osa,kenta\}@comm.ce.titech.ac.jp
}

\begin{abstract}
Obata et al. proved that spatially-coupled (SC) MacKay-Neal (MN) codes achieve the capacity of BEC. However, the SC-MN codes codes have many variable nodes of degree two and have higher error floors. In this paper, we prove that SC-MN codes with no variable nodes of degree two achieve the capacity of BEC.
\end{abstract}

\section{INTRODUCTION}

Felström and Zigangirov introduced spatially-coupled (SC) codes defined by sparse parity check matrix. SC codes are based on constitution method for convolutional LDPC codes [1]. Lantmaier et al. confirmed that regular SC LDPC codes achieve MAP threshold of original LDPC block codes by BP decoding in at least certain accuracy [2]. Kudekar et al. proved that $\mathrm{SC}$ codes achieve MAP threshold by BP decoding on binary erasure channel (BEC) [3] and binary symmetric channel [4].

Kasai et al. introduced SC MacKay-Neal (MN) codes, and showed that these codes with finite maximum degree achieve capacity of BEC by numerical experiment [5]. Obata et al. proved $(l, 2,2)$ SC-MN codes achieve capacity [6]. It has been observed that $(l, 2,2)$ SC-MN codes have many bit nodes of degree two. This leads to high error floors.

In this paper, we deal with $(l, 3,3) \mathrm{SC}-\mathrm{MN}$ codes whose bit node degree is greater than two. We prove the codes achieve the capacity of BEC. The codes achieve Shannon limit $\epsilon^{\text {Sha }}=$ $1-\frac{3}{l}$ for any $l \geq 3$.

\section{BACKGROUND}

\section{A. MacKay-Neal Codes}

$(l, r, g)$ MN codes are multi-edge type (MET) LDPC codes defined by pair of multi-variables degree distributions $(\mu, \nu)$ listed below.

$$
\begin{aligned}
\nu(\boldsymbol{x} ; \epsilon) & =\frac{r}{l} x_{1}^{l}+\epsilon x_{2}^{g}, \\
\mu(\boldsymbol{x}) & =x_{1}^{r} x_{2}^{g} .
\end{aligned}
$$

In general, the recursion of density evolution of MET-LDPC codes on BEC is given by

$$
y_{j}^{(t)}=1-\frac{\mu_{j}\left(\mathbf{1}-\boldsymbol{x}^{(t)} ; 1-\epsilon\right)}{\mu_{j}(\mathbf{1} ; 1)}, \quad x_{j}^{(t+1)}=\frac{\nu_{j}\left(\boldsymbol{y}^{(t)} ; \epsilon\right)}{\nu_{j}(\mathbf{1} ; 1)},
$$

where $x_{j}^{(t)}$ is probability of erasure message sent along edges of type $j$ at the $t$-th decoding round. Therefore, density evolution of $(l, r, g)$ MN codes is

$$
\begin{aligned}
& \boldsymbol{x}^{(t+1)}=\boldsymbol{f}\left(\boldsymbol{g}\left(\boldsymbol{x}^{(t)}\right) ; \epsilon\right), \\
& \boldsymbol{f}(\boldsymbol{x} ; \epsilon)=\left(x_{1}^{l-1}, \epsilon x_{2}^{g-1}\right), \\
& \boldsymbol{g}(\boldsymbol{x})=\left(1-\left(1-x_{1}\right)^{r-1}\left(1-x_{2}\right)^{g}, 1-\left(1-x_{1}\right)^{r}\left(1-x_{2}\right)^{g-1}\right) .
\end{aligned}
$$

\section{B. Spatially-Coupled MacKay-Neal Codes}

SC-MN codes of coupling number $L$ and of coupling width $w$ are defined by the Tanner graph constructed by the following process. First, at each section $i \in \mathbb{Z}$, place $r M / l$ bit nodes of type 1 and $M$ bits nodes of type 2. Bit nodes of type 1 and 2 are of degree $l$ and $g$, respectively. Next, at each section $i \in \mathbb{Z}$, place $M$ check nodes of degree $r+g$. Then, connect edges uniformly at random so that bit nodes of type 1 at section $i$ are connected with check nodes at each section $i \in[i, \ldots, i+w-1]$ with $r M / w$ edges, and bit nodes of type 2 at section $i$ are connected with check nodes at each section $i \in[i, \ldots, i+w-1]$ with $g M / w$ edges. Bits at section $i \notin[0, L-1])$ are shorten. Bits of type 1 and 2 at section $i \in[0, L-1]$ are punctured and transmitted, respectively. Rate of SC-MN codes $R^{M N}$ is given by

$$
R^{\mathrm{MN}}=\frac{r}{l}+\frac{1+w-2 \sum_{i=0}^{w}\left(1-\left(\frac{i}{w}\right)^{r+g}\right)}{L}=\frac{r}{l} \quad(L \rightarrow \infty) .
$$

\section{Vector Admissible System and Potential Function}

In this section, we define vector admissible systems and potential functions.

Definition 1. Define $\mathcal{X} \triangleq[0,1]^{d}$, and $F: \mathcal{X} \times[0,1] \rightarrow \mathbb{R}$ and $G: \mathcal{X} \rightarrow \mathbb{R}$ as functionals satisfying $G(\mathbf{0})=0$. Let $\boldsymbol{D}$ be a $d \times d$ positive diagonal matrix. Consider a general recursion defined by

$$
\boldsymbol{x}^{(t+1)}=\boldsymbol{f}\left(\boldsymbol{g}\left(\boldsymbol{x}^{(t)}\right) ; \epsilon\right)
$$

where $\boldsymbol{f}: \mathcal{X} \times[0,1] \rightarrow \mathcal{X}$ and $\boldsymbol{g}: \mathcal{X} \rightarrow \mathcal{X}$ are defined by $F^{\prime}(\boldsymbol{x} ; \epsilon)=\boldsymbol{f}(\boldsymbol{x} ; \epsilon) \boldsymbol{D}$ and $G^{\prime}(\boldsymbol{x})=\boldsymbol{g}(\boldsymbol{x}) \boldsymbol{D}$, where $F^{\prime}(\boldsymbol{x} ; \epsilon) \triangleq\left(\frac{\partial F(\boldsymbol{x})}{\partial x_{1}}, \ldots, \frac{\partial F(\boldsymbol{x})}{\partial x_{n}}\right)$. Then the pair $(\boldsymbol{f}, \boldsymbol{g})$ defines a vector admissible system if

1. $\boldsymbol{f}, \boldsymbol{g}$ are twice continuously differentiable,

2. $\boldsymbol{f}(\boldsymbol{x} ; \epsilon)$ and $\boldsymbol{g}(\boldsymbol{x})$ are non-decreasing in $\boldsymbol{x}$ and $\epsilon$ with respect to $\preceq 1$,

\footnotetext{
${ }^{1}$ We say $\boldsymbol{x} \preceq \boldsymbol{y}$ if $x_{i} \leq y_{i}$ for all $1 \leq i \leq d$
} 
3. $\boldsymbol{f}(\boldsymbol{g}(\mathbf{0}) ; \epsilon)=\mathbf{0}$ and $F(\boldsymbol{g}(\mathbf{0}) ; \epsilon)=0$.

We say $\boldsymbol{x}$ is a fixed point if $\boldsymbol{x}=\boldsymbol{f}(\boldsymbol{g}(\boldsymbol{x}) ; \epsilon)$.

It can be seen that the density evolution $(\boldsymbol{f}, \boldsymbol{g})$ of $(l, r, g)$ MN codes given in (2) is a vector admissible system by choosing $F(\boldsymbol{x} ; \epsilon), G(x)$ and $\boldsymbol{D}$ as below, since this system $(\boldsymbol{f}, \boldsymbol{g})$ satisfies the condition in Definition 1

$$
\begin{aligned}
F(\boldsymbol{x} ; \epsilon) & =\frac{r}{l} x_{1}^{l}+\epsilon x_{2}^{g}, \\
G(\boldsymbol{x}) & =r x_{1}+g x_{2}+\left(1-x_{1}\right)^{r}\left(1-x_{2}\right)^{g}-1, \\
\boldsymbol{D} & =\left(\begin{array}{ll}
r & 0 \\
0 & g
\end{array}\right) .
\end{aligned}
$$

Definition 2 ([7, Def. 2]). We define the potential function $U(\boldsymbol{x} ; \epsilon)$ of a vector admissible system $(\boldsymbol{f}, \boldsymbol{g})$ by

$$
U(\boldsymbol{x} ; \epsilon) \triangleq \boldsymbol{g}(\boldsymbol{x}) \boldsymbol{D} \boldsymbol{x}^{T}-G(\boldsymbol{x})-F(\boldsymbol{g}(\boldsymbol{x}) ; \epsilon) .
$$

The potential function $U\left(x_{1}, x_{2}, \epsilon\right)$ of $(l, r, g)$ MN codes is given by

$$
\begin{aligned}
U\left(x_{1}, x_{2}, \epsilon\right) & =1-\epsilon\left(\left(1-\left(1-x_{1}\right)^{r}\right)\left(1-x_{2}\right)^{g-1}\right)^{g} \\
& -\frac{r}{l}\left(1-\left(1-x_{1}\right)^{r-1}\left(1-x_{2}\right)^{g}\right)^{l} \\
& -\left(1-x_{1}\right)^{r}\left(1-x_{2}\right)^{g}\left(1+\frac{r x_{1}}{1-x_{1}}+\frac{g x_{2}}{1-x_{2}}\right) .
\end{aligned}
$$

Definition 3 ([7, Def. 7]). Let $\mathcal{F}(\epsilon) \triangleq\{\boldsymbol{x} \in \mathcal{X} \backslash\{\mathbf{0}\} \mid \boldsymbol{x}=$ $\boldsymbol{f}(\boldsymbol{g}(\boldsymbol{x}) ; \epsilon)\}$ be a set of non-zero fixed points for $\epsilon \in[0,1]$. The potential threshold $\epsilon^{*}$ is defined by

$$
\epsilon^{*} \triangleq \sup \left\{\epsilon \in[0,1] \mid \min _{\boldsymbol{x} \in \mathcal{F}(\epsilon)} U(\boldsymbol{x} ; \epsilon)>0\right\} .
$$

Let $\epsilon_{s}^{*}$ be threshold of uncoupled system defined in 7 Def. 6]. For $\epsilon$ such that $\epsilon_{s}^{*}<\epsilon<\epsilon^{*}$, we define energy gap $\Delta E(\epsilon)$ as

$$
\Delta E(\epsilon) \triangleq \max _{\epsilon^{\prime} \in[\epsilon, 1]} \inf _{\boldsymbol{x} \in \mathcal{F}\left(\epsilon^{\prime}\right)} U\left(\boldsymbol{x} ; \epsilon^{\prime}\right) .
$$

We define the SC system of a vector admissible system.

Definition 4 ([7, Def. 9]). For a vector admissible system $(\boldsymbol{f}, \boldsymbol{g})$, we define the SC system of coupling number $L$ and coupling width $w$ as

$$
\begin{aligned}
\boldsymbol{x}_{i}^{(t+1)} & =\frac{1}{w} \sum_{k=0}^{w-1} \boldsymbol{f}\left(\frac{1}{w} \sum_{j=0}^{w-1} \boldsymbol{g}\left(\boldsymbol{x}_{i+j-k}^{(t)}\right) ; \epsilon_{i-k}\right), \\
\epsilon_{i} & = \begin{cases}\epsilon, & i \in\{0, \ldots, L-1\}, \\
0, & i \notin\{0, \ldots, L-1\} .\end{cases}
\end{aligned}
$$

If we define $(\boldsymbol{f}, \boldsymbol{g})$ as the density evolution for $(l, r, g) \mathrm{MN}$ codes in (2), the SC system gives the density evolution of SC-MN codes with coupling number $L$ and coupling width $w$.

Next theorem states that if $\epsilon<\epsilon^{*}$ then fixed points of SC vector system converge towards $\mathbf{0}$ for sufficiently large $w$.

Theorem 1 ([7, Thm. 1]). Consider the constant $K_{f, g}$ defined in [7, Lem. 11]. This constant value depends only on $(\boldsymbol{f}, \boldsymbol{g})$. If $\epsilon<\epsilon^{*}$ and $w>\left(d K_{\boldsymbol{f}, \boldsymbol{g}}\right) /(2 \Delta E(\epsilon))$, then the SC system

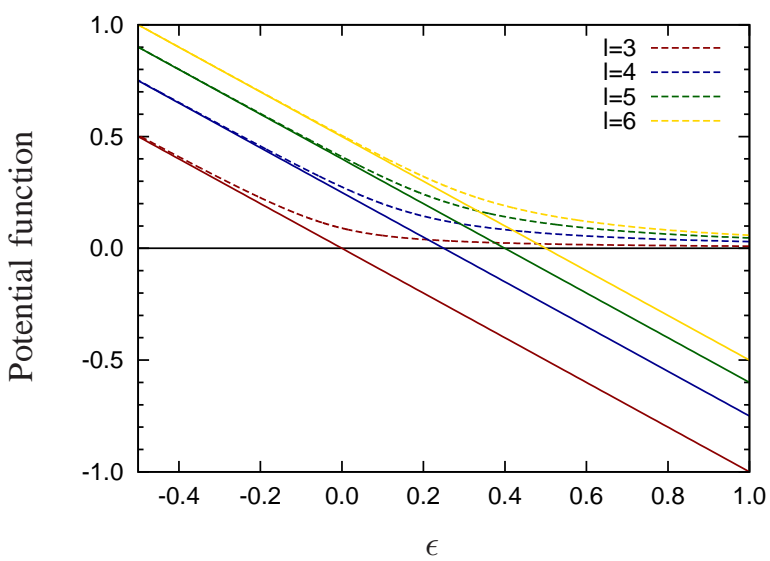

Fig. 1. Potential function $U(\mathbf{1} ; \epsilon)$ and $U\left(\boldsymbol{x}\left(x_{1}\right) ; \epsilon\left(x_{1}\right)\right)$ at the trivial fixed points (solid) and non-trivial fixed points (dashed) of $(l, 3,3) \mathrm{MN}$ codes for $l=3, \ldots, 6$

of $(\boldsymbol{f}, \boldsymbol{g})$ with coupling number $L$ and coupling width $w$ has a unique fixed point $\mathbf{0 .}$

We will show that the potential threshold $\epsilon^{*}$ of $(l, r=3, g=$ 3) $\mathrm{MN}$ codes is $1-R^{\mathrm{MN}}=1-3 / l$ for any $l \geq 3$. This is sufficient to show that $(l, 3,3)$ SC-MN codes with sufficiently large $w$ and $L$ achieve the capacity of BEC under BP decoding.

\section{PRoOF OF ACHIEVING CAPACITY}

In this section, we calculate the potential threshold $\epsilon^{*}$ of $(l, r=3, g=3) \mathrm{MN}$ codes. To this end, we first investigate the set of fixed points $\mathcal{F}(\epsilon)$.

The density evolution recursion in (2) can be rewritten as

$$
\begin{aligned}
& x_{1}^{(t+1)}=\left(1-\left(1-x_{1}^{(t)}\right)^{r-1}\left(1-x_{2}^{(t)}\right)^{g}\right)^{l-1}, \\
& x_{2}^{(t+1)}=\epsilon\left(1-\left(1-x_{1}^{(t)}\right)^{r}\left(1-x_{2}^{(t)}\right)^{g-1}\right)^{g-1} .
\end{aligned}
$$

Fixed points $\left(x_{1}, x_{2} ; \epsilon\right)$ of density evolution with $x_{1}=0$ and $x_{1}=1$ are $(0,0 ; \epsilon)$ and $(1, \epsilon ; \epsilon)$, respectively. We define these fixed points as trivial fixed points and all other fixed points as non-trivial fixed points. All non-trivial fixed points $\left(x_{1}, x_{2}\left(x_{1}\right) ; \epsilon\left(x_{1}\right)\right)$ can be parametrically described as

$$
\begin{aligned}
x_{2}\left(x_{1}\right) & =1-\left(\frac{1-x_{1}^{\frac{1}{l-1}}}{\left(1-x_{1}\right)^{r-1}}\right)^{\frac{1}{g}}, \\
\epsilon\left(x_{1}\right) & =\frac{x_{2}\left(x_{1}\right)}{\left(1-\left(1-x_{1}\right)^{r}\left(1-x_{2}\left(x_{1}\right)\right)^{g-1}\right)^{g-1}},
\end{aligned}
$$

with $x_{1} \in(0,1)$.

Next, we shall investigate the value of the potential function at the fixed points. The value of the potential functions at trivial fixed point $(1, \epsilon, \epsilon)$ is respectively given by

$$
U(1, \epsilon, \epsilon)=1-\frac{r}{l}-\epsilon .
$$

Figure 1 draws the potential function of $(l, r, g) \mathrm{MN}$ codes at fixed points $\boldsymbol{x} \in \mathcal{F}(\epsilon)$. It appears that the potential function at non-trivial fixed points is always positive. We will prove this. 
To be precise, the potential function of $(l, r, g) \mathrm{MN}$ codes for non-trivial fixed points satisfies

$$
U\left(x_{1}, x_{2}\left(x_{1}\right), \epsilon\left(x_{1}\right)\right)>0 \quad \text { for } \quad x_{1} \in(0,1) .
$$

Our strategy of proof is as follows. First change the representation of (4) into a polynomial form by changing variables a few times. Then apply Sturm's theorem for smaller $l$ and bound the polynomial for larger $l$.

We define $U(z):=\left.U\left(x_{1}, x_{2}\left(x_{1}\right), \epsilon\left(x_{1}\right)\right)\right|_{x_{1}=z^{l-1}}$. Obviously, to prove (4), it is sufficient to show $U(z)>0$ for $z \in(0,1)$.

$$
\begin{aligned}
U(z) & =-\frac{3 z^{l}}{l}+(1-z)\left(1-4 z^{l-1}\right) \\
& +(1-z)^{1 / 3}\left(1-z^{l-1}\right)^{-2 / 3}-2(1-z)^{2 / 3}\left(1-z^{l-1}\right)^{5 / 3} .
\end{aligned}
$$

We use next lemma to eliminate fractional power in $U(z)$. The proof is given in Section IV-A

Lemma 1. Define $H(u, z)$ as follows.

$$
\begin{aligned}
H(u, z) & =\left(u+\frac{3 z^{l}}{l}-(1-z)\left(1-4 z^{l-1}\right)\right)^{3} \\
& +6(1-z)\left(1-z^{l-1}\right)\left(u+\frac{3 z^{l}}{l}-(1-z)\left(1-4 z^{l-1}\right)\right) \\
& -(1-z)\left(1-z^{l-1}\right)^{-2}+8(1-z)^{2}\left(1-z^{l-1}\right)^{5} .
\end{aligned}
$$

Then, $H(0, z)<0$ for $z \in(0,1)$ implies $U(z)>0$ for $z \in$ $(0,1)$.

Define $I(z):=\frac{l^{3}\left(1-z^{l-1}\right)^{2}}{(1-z) z^{2}} H(0, z)$. Obviously, to prove $H(0, z)<0$ for $z \in(0,1)$, it is sufficient to prove $I(z)<0$ for $z \in(0,1)$. We see that $I(z)$ for $l \geq 3$ is a polynomial as follows.

$$
\begin{aligned}
I(z)= & -l^{3}+27 \sum_{i=0}^{l-2}\left[z^{3 l-2+i}\left(1-z^{l-1}\right)\right] \\
& -27 l^{2} z^{-2+2 l}\left(1-4 z^{l-1}\right)\left(1-z^{l-1}\right)^{2} \\
& -9 l z^{-4+l}\left(1-z^{l-1}\right)^{2}\left\{(-3+z) z^{2}\right. \\
& \left.+16(-1+z) z^{2 l}-8(-1+z) z^{1+l}\right\} \\
& -l^{3}(1-z) z^{-9+l}\left\{8 z^{6 l}-56 z^{1+5 l}+2 z^{6}(3+7 z)\right. \\
& +8 z^{2+4 l}(13+8 z)-8 z^{3+3 l}(13+22 z) \\
& \left.+4 z^{4+2 l}(21+43 z)-z^{5+l}(41+73 z)\right\} .
\end{aligned}
$$

We prove $I(z)<0$ for $3 \leq l<165$ and $l \geq 165$ in the following lemmas. The proofs are given in Section IV-B and Section IV-C respectively.

Lemma 2. For $3 \leq l<165, I(z)<0$ for $z \in(0,1)$.

Lemma 3. For $l \geq 165, I(z)<0$ for $z \in(0,1)$.

Theorem 2. For any $l \geq 3$ and $\epsilon<\epsilon^{\text {Sha }}=1-\frac{3}{l}$, the unique fixed point of density evolution of $(l, 3,3) S C-M N$ codes of coupling number $L$ and coupling width $w$ is $\mathbf{0}$ for sufficiently large $w$ and $L$.
Proof: From (4), potential function for non-trivial fixed points is always positive. Therefore, from Definition 3 and potential function for trivial fixed point (3), $\epsilon^{*}=1-\frac{r}{l}=\epsilon^{\text {Sha }}$. From Theorem 1 for $\epsilon<\epsilon^{\text {Sha }}$, the unique fixed point of density evolution for $(l, 3,3)$ SC-MN codes is $\mathbf{0}$.

The case with $l=3$ implies rate one codes over $\operatorname{BEC}(0)$. Some might think this is not interesting. Nevertheless, we included the case with $l=3$ for comprehensiveness.

\section{PROOF OF LEMMAS}

\section{A. Proof of Lemma $\square$}

Partial derivative of $H(u, z)$ with respect to $u$ gives

$$
\begin{aligned}
\frac{\partial H(u, z)}{\partial u}= & 3\left(u+\frac{3 z^{l}}{l}-(1-z)\left(1-4 z^{l-1}\right)\right)^{2} \\
& +6(1-z)\left(1-z^{l-1}\right) \geq 0
\end{aligned}
$$

Substituting $u=U(z)$ into $H(u, z)$ gives

$$
\begin{aligned}
H & (U(z), z) \\
= & \left((1-z)^{1 / 3}\left(1-z^{l-1}\right)^{-2 / 3}-2(1-z)^{2 / 3}\left(1-z^{l-1}\right)^{5 / 3}\right)^{3} \\
& +6(1-z)\left(1-z^{l-1}\right)\left\{(1-z)^{1 / 3}\left(1-z^{l-1}\right)^{-2 / 3}\right. \\
& \left.-2(1-z)^{2 / 3}\left(1-z^{l-1}\right)^{5 / 3}\right\} \\
& -(1-z)\left(1-z^{l-1}\right)^{-2}+8(1-z)^{2}\left(1-z^{l-1}\right)^{5} \\
= & (1-z)\left(1-z^{l-1}\right)^{-2}-8(1-z)^{2}\left(1-z^{l-1}\right)^{5} \\
& -6(1-z)\left(1-z^{l-1}\right)\left\{(1-z)^{1 / 3}\left(1-z^{l-1}\right)^{-2 / 3}\right. \\
& \left.-2(1-z)^{2 / 3}\left(1-z^{l-1}\right)^{5 / 3}\right\} \\
& +6(1-z)\left(1-z^{l-1}\right)\left\{(1-z)^{1 / 3}\left(1-z^{l-1}\right)^{-2 / 3}\right. \\
& \left.-2(1-z)^{2 / 3}\left(1-z^{l-1}\right)^{5 / 3}\right\} \\
& -(1-z)\left(1-z^{l-1}\right)^{-2}+8(1-z)^{2}\left(1-z^{l-1}\right)^{5} \\
= & 0
\end{aligned}
$$

From (6), $H(u, z)$ monotonically increasing with respect to $u$. From (7), $(u, z)=(U(z), z)$ is a root of $H(u, z)=0$. Therefore $H(0, z)<0$ for $z \in(0,1)$ implies $U(z)>0$ for $z \in(0,1)$.

\section{B. Proof of Lemma 2}

From $I(0)=-l^{3}$ and $I(1)=-l^{3}$, we see that $z=0,1$ are not multiple roots of equation $I(z)=0$. Let $I_{1}(z), \ldots, I_{m}(z)$ be Sturm sequences of $I(x)$. Let $V(z)$ be the number of sign changes in the sequence. Table $\square$ lists sign changes of Sturm sequence $I_{1}(z), \ldots, I_{m}(z)$ of $I(x)$ in (5) for $l=3, \ldots, 11$. $V(z)$ is the number of sign changes in the sequence. We see that $V(0)=V(1)$. We observed that $V(0)=V(1)$ for $l<165$ but not listed all due to the space limit. From Theorem 3 this implies that the number of distinct roots of equation $I(z)=0$ in $(0,1]$ is $V(0)-V(1)=0$. Therefore, $I(z)<0, z \in(0,1)$ for $3, \ldots, 164$. 
TABLE I

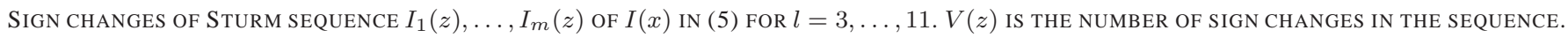

\begin{tabular}{|c|c|c|c|c|}
\hline & $m$ & $V(z)$ & $z$ & $\operatorname{sgn}\left[I_{0}(z)\right], \operatorname{sgn}\left[I_{1}(z)\right], \ldots, \operatorname{sgn}\left[I_{m}(z)\right]$ \\
\hline 4 & & 10 & 1 & $\begin{array}{l}--+---+---++-+++--+--- \\
--+---+--++----+--+--\end{array}$ \\
\hline \multirow[t]{2}{*}{6} & 33 & 16 & 0 & $-0+---++-+++--++--+++-++--++--+---$ \\
\hline & & 16 & 1 & --++----+-++----+++---++-+---+-+++- \\
\hline 8 & 45 & $\begin{array}{l}22 \\
22\end{array}$ & $\begin{array}{l}0 \\
1\end{array}$ & \\
\hline \multirow[t]{2}{*}{9} & 51 & 24 & 0 & $-0+---++--++--+-+++++-+++--++---+--++---+--+----+++-$ \\
\hline & & 24 & 1 & --++---++--++-+--+++++--+++--+++-++-+++-++--+++-+++- \\
\hline \multirow[t]{2}{*}{10} & 57 & 28 & 0 & $-0+---++---+-+++-+----++---++-+++---++-+---++-++-++++-+++-$ \\
\hline & & 28 & 1 & --++---+++--+-++--+-----++-+++--+++---+-+---++-++---+--++- \\
\hline 11 & 63 & $\begin{array}{l}30 \\
30\end{array}$ & $\begin{array}{l}0 \\
1\end{array}$ & 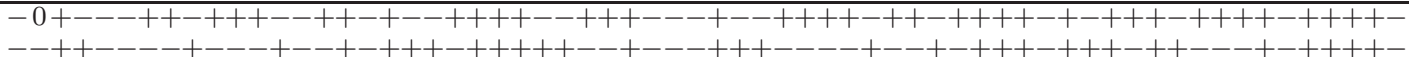 \\
\hline
\end{tabular}

\section{Proof of Lemma 3}

We first claim that for $z \in(0,1)$,

$$
\begin{aligned}
& \text { If } \frac{a l+b}{a l+b+1} \in(0,1) \text {, then } \\
& q(z):=z^{a l+b}(1-z) \leq \frac{1}{a l+b+1}, \\
& \text { If } \frac{a l+b}{(a+2) l+b-2} \in(0,1), \text { then } \\
& r(z):=z^{a l+b}\left(1-z^{l-1}\right)^{2} \leq\left(\frac{2 l-2}{(a+2) l+b-2}\right)^{2}
\end{aligned}
$$

Differentiating $q(z)$ gives

$$
\frac{\mathrm{d} q(z)}{\mathrm{d} z}=z^{a l+b-1}(a l+b-(a l+b+1) z) .
$$

Since $\frac{a l+b}{a l+b+1} \in(0,1)$, we see that $z=\frac{a l+b}{a l+b+1}$ gives the maximum value of $q(z)$.

$$
q(z) \leq\left(\frac{a l+b}{a l+b+1}\right)^{a l+b} \frac{1}{a l+b+1}<\frac{1}{a l+b+1} .
$$

Differentiating $r(z)$ gives

$\frac{\mathrm{d} r(z)}{\mathrm{d} z}=z^{a l+b-1}\left(1-z^{l-1}\right)\left((a l+b)-((a+2) l+b-2) z^{l-1}\right)$

Since $\frac{a l+b}{(a+2) l+b-2} \in(0,1)$, we see that $z=\left(\frac{a l+b}{(a+2) l+b-2}\right)^{\frac{1}{l-1}}$ gives the maximum value of $r(z)$. Thus, next inequality holds.

$$
\begin{aligned}
r(z) & \leq\left(\frac{a l+b}{(a+2) l+b-2}\right)^{\frac{a l+b}{l-1}}\left(\frac{2 l-2}{(a+2) l+b-2}\right)^{2} \\
& <\left(\frac{2 l-2}{(a+2) l+b-2}\right)^{2} .
\end{aligned}
$$

In (a), we eliminate negative terms except for $-l^{3}$. Next, in (b), we apply (8) and (9) to each term of (5) by using $l \geq 165$.
We obtain an upper bound of $I(z)$ for $z \in(0,1)$ as follows.

$$
\begin{aligned}
I(z) \stackrel{(\mathrm{a})}{<} & -l^{3}+27 \sum_{i=0}^{l-2} z^{3 l-2+i}\left(1-z^{l-1}\right) \\
& +108 l^{2} z^{-3+3 l}\left(1-z^{l-1}\right)^{2} \\
& +9 l z^{-4+l}\left(1-z^{l-1}\right)^{2}\left(3 z^{2}+16 z^{2 l}+8 z^{2+l}\right) \\
& +l^{3}\left\{( 1 - z ) z ^ { - 9 + l } \left[56 z^{1+5 l}+8 z^{3+3 l}(13+22 z)\right.\right. \\
& \left.\left.+z^{5+l}(41+73 z)\right]\right\} \\
(\mathrm{b}) & -l^{3}+27 \sum_{i=0}^{l-2}[1]+108 l^{2}\left(\frac{2 l-2}{5 l-5}\right)^{2} \\
& +9 l\left(3\left(\frac{2 l-2}{3 l-4}\right)^{2}+16\left(\frac{2 l-2}{5 l-6}\right)^{2}+8\left(\frac{2 l-2}{4 l-4}\right)^{2}\right) \\
& +l^{3}\left(\frac{56}{6 l-7}+\frac{176}{4 l-4}+\frac{104}{4 l-5}+\frac{41}{2 l-3}+\frac{73}{2 l-2}\right)
\end{aligned}
$$

$$
\begin{aligned}
\stackrel{(\mathrm{c})}{<} & -l^{3}+27(l-1)+\frac{432 l^{2}}{25}+9 l\left(3 \frac{5}{9}+16 \frac{1}{5}+8 \frac{1}{4}\right) \\
& +5 l^{3}\left(\frac{59}{29 l}+\frac{176}{19 l}+\frac{104}{19 l}+\frac{73}{9 l}+\frac{41}{9 l}\right) \\
< & -l^{3}+\frac{6775346}{41325} l^{2}+\frac{444}{5} l=: \bar{I}(l) .
\end{aligned}
$$

.We used next inequalities valid for $l \geq 165$ in (c).

$$
\begin{array}{rlrl}
\left(\frac{2 l-2}{3 l-4}\right)^{2} & \leq \frac{5}{9}, & & \left(\frac{2 l-2}{5 l-6}\right)^{2} \leq \frac{1}{5}, \\
6 l-7 & \geq \frac{29 l}{5}, & 4 l-4 & \geq \frac{19 l}{5}, \\
4 l-5 & \geq \frac{19 l}{5}, & 2 l-3 & \geq \frac{9 l}{5}, \\
2 l-2 & \geq \frac{9 l}{5} . &
\end{array}
$$

The roots of $\bar{I}(l)=0$ are 0 and $\frac{3387673 \pm \sqrt{11627977054429}}{41325} \simeq$ $-0.53984,+164.49$. Thus, we conclude that for $l \geq 165$ and $z \in(0,1), I(z)<\bar{I}(l)<0$. 


\section{CONCLusion And Future Work}

In this paper, we proved that $(l, 3,3) \mathrm{SC}-\mathrm{MN}$ codes with $l \geq 3$ achieve capacity on the BEC under BP decoding for sufficiently large $L$ and $w$. This codes do not have bit nodes of degree two and have low error floors. We proved that the potential threshold and Shannon limit of $(l, r=3, g=3) \mathrm{MN}$ codes on the BEC are the same.

\section{REFERENCES}

[1] A. J. Felström and K. S. Zigangirov, "Time-varying periodic convolutional codes with low-density parity-check matrix," IEEE Trans. Inf. Theory, vol. 45, no. 6, pp. 2181-2191, June 1999.

[2] M. Lentmaier, D. V. Truhachev, and K. S. Zigangirov, "To the theory of low-density convolutional codes. II," Probl. Inf. Transm., no. 4, pp. 288-306, 2001.

[3] S. Kudekar, T. Richardson, and R. Urbanke, "Threshold saturation via spatial coupling: Why convolutional LDPC ensembles perform so well over the BEC," IEEE Trans. Inf. Theory, vol. 57, no. 2, pp. 803-834, Feb. 2011.

[4] - " "Spatially coupled ensembles universally achieve capacity under belief propagation," IEEE Trans. Inf. Theory, vol. 59, no. 12, pp. 77617813, 2013.

[5] K. Kasai and K. Sakaniwa, "Spatially-coupled MacKay-Neal codes and Hsu-Anastasopoulos codes," IEICE Trans. Fundamentals, vol. E94-A, no. 11, pp. 2161-2168, Nov. 2011.

[6] N. Obata, Y.-Y. Jian, K. Kasai, and H. D. Pfister, "Spatially-coupled multiedge type LDPC codes with bounded degrees that achieve capacity on the BEC under BP decoding," in Proc. 2013 IEEE Int. Symp. Inf. Theory (ISIT), July 2013, pp. 2433-2437.

[7] A. Yedla, Y.-Y. Jian, P. Nguyen, and H. Pfister, "A simple proof of threshold saturation for coupled scalar recursions," in Turbo Codes and Iterative Information Processing (ISTC), 2012 7th International Symposium on, 2012, pp. 51-55.

[8] W. Gautschi, Numerical Analysis, ser. SpringerLink : Bücher. Springer Science+Business Media, LLC, 2011.

\section{APPENDIX STURM'S THEOREM}

Theorem $3([[8])$. For a polynomial $f(x)$ over $\mathbb{R}$, we define Sturm sequences $f_{i}(x)(i=0, \ldots, m)$ as $f(x), f^{\prime}(x)$ and polynomials obtained by applying Euclid's algorithm to $f(x)$ and $f^{\prime}(x)$.

$$
\begin{aligned}
f_{0}(x) & =f(x), \\
f_{1}(x) & =f^{\prime}(x), \\
f_{n-1}(x) & =q_{n}(x) f_{n}(x)-f_{n+1}(x) \quad(n=1, \ldots, m-1), \\
f_{m-1}(x) & =q_{m}(x) f_{m}(x) .
\end{aligned}
$$

For real number $c$, let $V(c)$ be the number of sign changes in $f_{0}(c), f_{1}(c), \ldots, f_{m}(c)$. If neither $a \in \mathbb{R}$ nor $b \in \mathbb{R}$ is a multiple root of $f(x)=0$, then the number of distinct roots of $f(x)$ in $(a, b]$ is $V(a)-V(b)$. 